\title{
Adjustments to recent and frequent conflict reflect two distinguishable mechanisms
}

\author{
Sascha Purmann \\ Ruprecht Karls University Heidelberg, Heidelberg, Germany \\ AND \\ Stephanie Badde and Mike Wendt \\ Helmut Schmidt University, Hamburg, Germany \\ and University of the Federal Armed Forces, Hamburg, Germany
}

\begin{abstract}
In conflict paradigms such as the Eriksen flanker task, interference has been found to be reduced under conditions of recent and/or frequent cognitive conflict. Using a modified flanker task, we investigated the interplay of conflict recency and conflict frequency by comparing the interference reductions following conflict trials under conditions in which conflict was either frequent or infrequent overall, while controlling for stimulus and response feature repetitions to rule out nonattentional accounts. The reduction of flanker interference after a conflict trial was attenuated when overall conflict was frequent. This result is consistent with models assuming that processing adjustments occur gradually in response to conflict strength, such as the connectionist model of Botvinick, Braver, Barch, Carter, and Cohen (2001), in which both recency and frequency modulations are brought about by the same mechanism. By decomposing response times into initiation times and movement times, we revealed that frequent conflict delayed response initiation but sped up movement. Moreover, whereas frequent conflict reduced interference in both components, interference reduction after individual conflict trials was confined to movement times. Taken together, these results suggest that different mechanisms underlie the two kinds of modulation.
\end{abstract}

Human information processing is selective. Our cognitive system somehow favors information that is relevant to the achievement of current goals. Nevertheless, selectivity is far from being perfect, as has been shown in experimental tasks used to study selective attention, such as the Stroop task (Stroop, 1935), the Simon task (Simon, Craft, \& Small, 1971), and the Eriksen task (Eriksen \& Eriksen, 1974).

In the Eriksen flanker task, participants discriminate between target stimuli, such as the letter $H$ or $S$, presented in the center of four homologous distractor stimuli, two on each side of the target stimulus. In this task, response times (RTs) and error proportions are usually greater on trials in which the target and flankers are associated with different responses (e.g., HHSHH) rather than the same response (e.g., SSSSS). In the following report, we refer to a stimulus as compatible when the target and distractors call for the same response and as incompatible when they call for different responses. We refer to the performance difference between these two categories of stimuli as the flanker effect.

Interference effects are influenced by the frequency and recency of compatible and incompatible stimuli. The flanker effect is reduced when the proportion of incompatible stimuli increases (see, e.g., Gratton, Coles, $\&$ Donchin, 1992). Similar interference reductions have been obtained when the directly preceding trial consists of an incompatible rather than a compatible stimulus (e.g., Ullsperger, Bylsma, \& Botvinick, 2005). Both frequency modulation and recency modulation have also been found in other paradigms, such as Stroop-like tasks (e.g., Kerns et al., 2004; Logan \& Zbrodoff, 1979) or the Simon task (Hommel, 1994; Stürmer, Leuthold, Soetens, Schröter, \& Sommer, 2002), thus indicating broader generality of the underlying mechanisms.

Current models of cognitive control account for both findings by assuming that attentional adjustment occurs in response to cognitive conflict. Most prominently, the connectionist model of Botvinick, Braver, Barch, Carter, and Cohen (2001) accounts for response conflict as concurrent activation of divergent response units, measured as Hopfield energy, and cognitive control as preactivation of stimulus input units, concentrated more or less on the target location. Conflict in this model influences the amount of cognitive control occurring in the following trial, as expressed in the following equation:

$$
C(n+1)=\lambda C(n)+(1-\lambda)[\alpha E(n)+\beta] .
$$

$C(n+1)$ here represents cognitive control in trial $n+1$, which is a function of $C(n)$, the amount of control in trial $n$, and $E(n)$, the level of conflict in trial $n$, such that control in

S. Purmann, sascha.purmann@psychologie.uni-heidelberg.de 
trial $n+1$ is increased after a conflict trial and decreased after a nonconflict trial. $E(n)$, on the other hand, depends on $C(n)$ - that is, the higher the control in a trial, the lower the conflict. The parameters $\alpha$ and $\beta$ are scaling parameters, and $\lambda$ weights the influence of conflict in trial $n$. Two things follow from this equation. First, with each incompatible trial, cognitive control is increased (until a maximum amount is finally reached), so that cognitive control is higher on average when incompatible trials are frequent, and hence the flanker effect is smaller (frequency modulation). In this model, therefore, both frequency modulation and recency modulation can be accounted for by the same mechanism. Second, less adaptation to conflict will occur after an incompatible trial that is preceded by an incompatible trial than after an incompatible trial that is preceded by a compatible trial. This is because after an incompatible trial, cognitive control is increased, allowing less conflict to occur and, as a consequence, less adaptation. Since sequences of two or more incompatible trials occur more often in task blocks in which incompatible trials are frequent than in blocks in which they are infrequent, recency modulation should be weaker under these conditions. In line with this reasoning, Ridderinkhof (2002) found stronger adjustments of processing strategy after committing an error in the Simon task when the overall frequency of conflict trials decreased. Also, Stürmer et al. (2002, Experiment 1) analyzed the interplay of conflict frequency and recency in a Simon task. Although the strength of the recency effect was not formally tested in that experiment, the effect did seem to differ between conditions of low (20\%) and high (80\%) conflict frequency, and was more pronounced under both of these conditions than when conflict frequency was at an intermediate level $(50 \%)$. However, because the trial-to-trial sequence of compatibility levels in that experiment was perfectly confounded with the sequence of stimulus (location) and response features, the degree to which the recency effect obtained was brought about by selectivity adjustment or event file retrieval cannot be decided (for a more detailed discussion of this issue, see below).

It is important to note that the prediction of stronger recency modulation under conditions of frequent conflict is not unique to models that account for both frequency and recency modulations by the same mechanism. An alternative interpretation was suggested by Stürmer et al. (2002). Given that distractors co-occur with certain responses under conditions of frequent conflict, these authors assumed that frequency modulation reflects associative distractor-response priming (which facilitates performance in incompatible trials when conflict is frequent and in compatible trials when conflict is rare) rather than an adjustment of processing selectivity. Obtaining a stronger recency effect under conditions of infrequent conflict might also be consistent with this two-mechanism approach, if it is assumed that the selectivity adjustment is related to the degree of response conflict by means of associative flanker-response priming. Put more generally, inasmuch as the degree of selectivity adjustment is related to the strength of conflict, any manipulation that decreases the conflict exerted by an incompatible stimu- lus (on a directly preceding trial) should lead to weaker recency modulation.

Although obtaining weaker recency modulation under conditions of increased conflict frequency would thus not enable us to distinguish between models in which recency and frequency modulations are accounted for either by one mechanism or by two dependent mechanisms, this finding would still be important, since both models predict this interaction. In addition, such a finding would allow us to reject any model that proposed two independent mechanisms, although to our knowledge no such model has ever been introduced in the literature.

To examine the interplay of conflict recency and conflict frequency, we varied the overall proportion of trials with compatible and incompatible flankers between two groups of participants and analyzed the flanker effect as a function of both conflict frequency/group and the compatibility level of the stimulus on the directly preceding trial-that is, both frequency and recency modulations occurred in a single experiment.

When investigating the recency modulation, it is important to control for the sequence of stimulus features (e.g., targets and flankers) and of responses. In standard paradigms (i.e., using limited sets of stimuli and responses), repetitions of the compatibility level (i.e., a compatible trial following a compatible trial or an incompatible trial following an incompatible trial) tend to be associated with either repetition or alternation of both the target and the flankers. Compatibility level alternations, on the other hand, tend to be associated with repetition of either the target or the flankers and alternation of the other feature (i.e., partial feature repetition). According to Hommel's (1998) event file theory, partial feature repetitions are associated with a processing disadvantage because of a mismatch between the prior processing episode and the current task demands. It is therefore possible that reductions of the flanker effect after a conflict trial reflect such processing disadvantages rather than an adjustment of selectivity (Hommel, Proctor, \& Vu, 2004). To control for the effect of feature sequences, we applied a 4:4 mapping between (target) stimuli and responses and included in the statistical analyses only trials in which all stimulus and response features differed from those in the preceding trial (Ullsperger et al., 2005).

Participants pressed any of four possible responses with the same finger, which was placed on a central rest button at the beginning of each trial. This arrangement made it possible to decompose RTs into the time required to release the rest button (initiation times, ITs) and the time required to move the finger to the target button (movement times, MTs), and thus to determine the flanker effect — and any modulations thereof - for each component separately. Such an analysis is interesting for at least two reasons. First, it allows us to examine the temporal dynamics of adaptation to recent and frequent conflict. If these adaptations affect early stages of information processing, effects should already be evident in the ITs. Second, it can potentially provide information to differentiate the models described above. Assuming that recency and frequency modulations go back to one mechanism, one effect should 
appear in ITs only if the other effect appears at that stage as well, and not appear if the other effect does not appear. The same reasoning applies to MTs.

To summarize, we first examined the interplay of flanker effect modulations in RTs in response to frequent and recent conflict, in order to test the predictions derived from models proposing one mechanism of adaptation to conflict or two dependent mechanisms, as well as to differentiate between these models and models proposing two independent mechanisms. We then examined ITs and MTs in order to differentiate between the one-mechanism and two-dependent-mechanisms models.

\section{METHOD}

\section{Participants}

Fourteen students ( 8 female and 6 male, age range $21-32$ years) at the University of Hamburg participated in the infrequent-conflict group. Another 14 students ( 8 female and 6 male, age range 18-27 years) at Ruprecht Karls University Heidelberg participated in the frequent-conflict group. All participants had normal or corrected-to-normal vision and were naive with respect to the purpose of the study. Some participated for partial course fulfillment.

\section{Apparatus and Stimuli}

Presentation software (version 10.1, available from www.neurobs .com) was used for stimulus presentation and response recording on an IBM-compatible PC. Landolt rings (broken circles commonly used as test objects to evaluate visual acuity) with a gap at the top, left, bottom, or right were used as the stimuli. The target stimulus was presented centrally and was flanked by single distractor rings on each of four sides (top, left, bottom, and right; all distractors were identical with regard to the position of the gap). All stimuli, instructions, and feedback were presented in black on a white background on a 17 -in. CRT monitor. The participants made fast, discriminative responses with their right index finger on a box with four response buttons (arranged in a cross-like fashion) and a centrally positioned button (Cedrus response box, model RB-520; San Pedro, CA). The participants had to press the response button corresponding to the gap in the target ring. All responses were started from the central button, so that three response measures were recorded: response IT (i.e., the time when the central button was released), total RT (i.e., the time when one of the four response buttons was pressed), and MT (i.e., the difference between the total RT and the IT). Participants were instructed to make responses as fast as possible while maintaining an error rate of less than $10 \%$.

\section{Procedure}

Each trial comprised the following events: a fixation cross at the center of the screen for $400 \mathrm{msec}$, a blank screen for $600 \mathrm{msec}$, the flankers presented alone for $100 \mathrm{msec}$, and the target and flankers for another $100 \mathrm{msec}$. There was no time limit for participant responses. After each response, the screen remained blank for $600 \mathrm{msec}$, during which time participants had to return their finger to the central button. The ratios of incompatible to compatible trials were 25:75 for the infrequent-conflict group and 75:25 for the frequent-conflict group. The stimuli were chosen randomly within these constraints, and participants completed 13 blocks of 100 trials each. To further encourage the adoption of "infrequent-conflict" and "frequent-conflict" strategies, 4 additional trials were presented to each group both before and after the 100 trials per block; these trials were uniformly compatible for the infrequent-conflict group and incompatible for the frequent-conflict group (cf. Kerns et al., 2004). These added trials were not included in the data analyses. After each block, performance feedback was provided in the form of a participant's mean RT and error rate. Participants started each block at a self-determined point in time.

\section{RESULTS}

The first block was considered training and thus was not analyzed. Trials in which participants did not start from the rest button were excluded from further analysis as invalid trials. This was the case for $3.03 \%$ of all trials in the infrequent-conflict group and $3.25 \%$ in the frequent-conflict group. The data analyses were confined to trials in which all stimulus features (i.e., target and flankers) changed with respect to the preceding trial (on average, $65 \%$ and $35 \%$ of all trials for the infrequent- and frequent-conflict groups, respectively). Note that this rule also excluded trials in which the target had appeared as the flankers on the preceding trial (and vice versa). Trials with ITs of less than $100 \mathrm{msec}$, MTs of less than $30 \mathrm{msec}$, or RTs of more than 1,200 msec were also excluded from the analysis (less than $1 \%$ of all trials). The error rate was calculated as the relative frequency of error trials among all of the remaining trials. Both error trials and the trials that followed them $(2.27 \%$ and $1.83 \%$ of all trials, respectively) were excluded from further analyses. The error rates were too low to allow statistical analyses but are reported in Table 1. On average, these criteria resulted in the numbers of observations per participant and condition shown in Table 1. All analyses were carried out using $\mathrm{R}$ (version 2.5.1; R Development Core Team, 2007). Statistical modeling was conducted stepwise: First, a linear mixed model with main effects only was assessed. To test all main effects separately for significance, we deleted the term for each effect from the model and compared each of the resulting simpler models with the initial model. We then augmented the initial model with the interaction terms based on our hypotheses and tested these terms for significance by comparing the extended models with the next simpler model, starting with the initial model. The parameters of the models were estimated using the maximum likelihood procedure in R's nonlinear and linear mixed effect model package (Pinheiro \& Bates, 2000). In contrast to a conventional analytical approach (e.g., ANOVAs), this method allows for robust estimates even when the design is unbalanced. Furthermore, instead of assuming homogeneous variances, a more appropriate variance structure could be incorporated into the model. Since variance normally increases

Table 1

Number of Observations (per Participant and Condition), Mean Response Times (RTs), Mean Initiation Times (ITs), Mean Movement Times (MTs), and Error Rates (ERs) As Functions of Conflict Frequency, Compatibility on the Preceding Trial, and Compatibility on the Current Trial

\begin{tabular}{lrrrrr}
\hline $\begin{array}{c}\text { Preceding Trial/ } \\
\text { Current Trial }\end{array}$ & $n$ & $\begin{array}{c}\text { RT } \\
(\mathrm{msec})\end{array}$ & $\begin{array}{c}\text { IT } \\
(\mathrm{msec})\end{array}$ & $\begin{array}{c}\text { MT } \\
(\mathrm{msec})\end{array}$ & $\begin{array}{c}\text { ER } \\
(\%)\end{array}$ \\
\hline \multicolumn{7}{c}{ Infrequent-Conflict Group } \\
Compatible/compatible & 393 & 381 & 262 & 119 & 0.1 \\
Incompatible/compatible & 127 & 389 & 272 & 118 & 0.1 \\
Compatible/incompatible & 128 & 477 & 300 & 177 & 3.6 \\
Incompatible/incompatible & 21 & 479 & 314 & 166 & 1.8 \\
\multicolumn{7}{c}{ Frequent-Conflict Group } \\
Compatible/compatible & 49 & 488 & 388 & 100 & 0.1 \\
Incompatible/compatible & 102 & 494 & 392 & 102 & 0.1 \\
Compatible/incompatible & 99 & 544 & 413 & 131 & 2.1 \\
Incompatible/incompatible & 97 & 547 & 420 & 127 & 1.5 \\
\hline
\end{tabular}


Table 2

Model Comparisons for Response Time Models

\begin{tabular}{|c|c|c|c|c|c|c|}
\hline Model & $d f$ & $\mathrm{AIC}$ & $\begin{array}{l}\text { Log } \\
\text { Lik. }\end{array}$ & Test & $\begin{array}{l}\text { Lik. } \\
\text { Ratio }\end{array}$ & $p$ \\
\hline $1 \beta_{1}+\beta_{2}+\beta_{3}$ & 9 & $-19,679$ & 9,848 & & & \\
\hline $2 \beta_{2}+\beta_{3}$ & 8 & $-19,666$ & 9,841 & 1 vs. 2 & 14.6 & $<.001$ \\
\hline $3 \beta_{1}+\beta_{3}$ & 8 & $-19,633$ & 9,825 & 1 vs. 3 & 47.2 & $<.001$ \\
\hline $4 \beta_{1}+\beta_{2}$ & 8 & $-16,668$ & 8,342 & 1 vs. 4 & $3,012.7$ & $<.001$ \\
\hline $5 \beta_{1}+\beta_{2}+\beta_{3}+\beta_{4}$ & 10 & $-20,358$ & 10,189 & 1 vs. 5 & 681.1 & $<.001$ \\
\hline $6 \beta_{1}+\beta_{2}+\beta_{3}+\beta_{4}+\beta_{5}$ & 11 & $-20,373$ & 10,198 & 5 vs. 6 & 17.0 & $<.001$ \\
\hline $7 \beta_{1}+\beta_{2}+\beta_{3}+\beta_{4}+\beta_{5}+\beta_{6}$ & 13 & $-20,382$ & 10,204 & 6 vs. 7 & 13.2 & $<.002$ \\
\hline $8 \begin{array}{l}\beta_{1}+\beta_{2}+\beta_{3}+\beta_{4}+\beta_{5}+\beta_{6} \\
\text { (homogeneous variances) }\end{array}$ & 10 & $-19,705$ & 9,862 & 7 vs. 8 & 683.6 & $<.001$ \\
\hline
\end{tabular}

with increasing RTs, we allowed for different variances for responses to compatible and incompatible stimuli and for variances to vary between groups with respect to the two stimulus categories. Also, we did not aggregate data per participant and condition but worked with the individual trial data, an approach known as multilevel modeling. Because distributions of RTs, ITs, and MTs were skewed, we used the log-transformed measures as the response variables in the modeling process.

\section{Response Times}

Average RTs are reported in Table 1. First, we assessed the following initial model:

$$
y_{i j k m}=\beta_{1 j}+\beta_{2 k}+\beta_{3 m}+b_{i}+e_{i j k m},
$$

where $\beta_{1 j}$ represents the main effect of conflict frequency $(j \in\{0,1\}), \beta_{2 k}$ the effect of conflict in trial $n-1(k \in$ $\{0,1\}), \beta_{3 m}$ the effect of conflict in trial $n(m \in\{0,1\})$, and $b_{i}$ the random effects for the participants $(i \in\{0, \ldots$, $28\})$. It is assumed that $b_{i}$ and the residual errors $e_{i j k m}$ are normally distributed around zero with unknown standard deviations, $\sigma_{b}$ and $\sigma_{e}$. To test for a main effect of conflict frequency, we compared our initial model with a simpler one that did not include the $\beta_{1 j}$ term. Our initial model fitted the data better, as measured by the Akaike information criterion (AIC; Akaike, 1973) of -19,679, as compared with $-19,666$ for the simpler model, and the likelihood ratio of $\chi^{2}(1)=14.6, p<.001$. The AIC penalizes model complexity, so simpler models are better according to this measure, as long as the more complex model does not lead to a substantial improvement of fit. Following the same logic, we built a second model by excluding $\beta_{2 k}$, and a third by excluding $\beta_{3 m}$ from the initial model. In both cases, our initial model fitted the data better (see Table 2 for details), indicating that RTs in the frequent-conflict group were shorter overall (518 vs. $432 \mathrm{msec}$ ), that RTs were longer for incompatible stimuli in trial $n$ (512 vs. $438 \mathrm{msec}$ ), and that RTs were shorter following incompatible stimuli in trial $n-1$ (477 vs. $472 \mathrm{msec}$ ). Therefore, all three main effects were significant. To test for an interaction of conflict frequency and conflict in trial $n$, we extended the initial model by a corresponding interaction term $\left(\beta_{4}\right)$. Inclusion of this interaction term increased model fit $\left[\chi^{2}(2)=681.1\right.$, $p<.001]$ : The flanker effect was significantly larger in the infrequent-conflict group (93 vs. $55 \mathrm{msec}$ ). To test for an interaction of conflict in the current and in the previous trial (recency effect), we extended this model by a corresponding interaction term $\left(\beta_{5}\right)$. Model fit again increased $\left[\chi^{2}(1)=17.0, p<.001\right]$; that is, the flanker effect was reduced after incompatible trials ( $76 \mathrm{vs} .71 \mathrm{msec})$. Finally, to test for an interaction of both interference modulations, we included this interaction term in the model $\left(\beta_{6}\right)$. This further increased model fit $\left[\chi^{2}(2)=13.19, p=.0014\right]$; that is, the recency modulation also depended on conflict frequency, in that the reduction of interference after conflict in the preceding trial was greater in the infrequentconflict group than in the frequent-conflict group (6 vs. $3 \mathrm{msec}$ ). To test our assumption of heterogeneous variance, we compared the last model (for which variances were allowed to differ for compatible and incompatible stimuli and for each group) with a model assuming homoscedasticity. This comparison confirmed that the heteroscedastic model fit the data better $\left[\chi^{2}(3)=683.6, p<.001\right]$. Note that in all cases, these results were supported by the AIC (see Table 2).

To further examine interference reductions under conditions of frequent and recent conflict processing, we analyzed release times of the rest button (i.e., response initiation) and MTs separately. Note that this analysis is exploratory. We chose the best-fitting model through a stepwise elimination procedure using the stepAIC function of R's MASS package. This algorithm eliminates one term at a time from the full model (i.e., the one including all main effects and interactions) and stops when the AIC of the model increases, in this way identifying the most parsimonious model.

\section{Response Initiation Times}

Average ITs are reported in Table 1. The most parsimonious model (identified by stepwise elimination of terms from the full model) included the terms conflict frequency, conflict in trial $n-1$, conflict in trial $n$, the interaction between conflict frequency and conflict in trial $n-1$, and the interaction between conflict frequency and conflict in trial $n$. The ANOVAs for this model are given in Table 3. We can conclude from the model that all main effects were significant. ITs were faster overall in the infrequent-conflict group ( $287 \mathrm{vs.} 403 \mathrm{msec}$ ), faster for compatible stimuli (328 vs. $361 \mathrm{msec}$ ), and slower after incompatible stimuli in trial $n-1$ (341 vs. $349 \mathrm{msec}$ ). Furthermore, the flanker effect was smaller in the frequent-conflict group ( $27 \mathrm{vs.} 40 \mathrm{msec}$ ), and the in- 
Table 3

ANOVA Table for the Most Parsimonious Response Initiation Time Model

\begin{tabular}{llccrc}
\hline \multicolumn{1}{c}{ Term } & $d f_{\text {num }}$ & $d f_{\text {den }}$ & \multicolumn{1}{c}{$F$} & $p$ \\
\hline 1 & Group & 1 & 14054 & 52.2 & $<.001$ \\
2 & Conflict in trial $n-1$ & 1 & 14054 & 92.6 & $<.001$ \\
3 & Conflict in trial $n$ & 1 & 14054 & 509.1 & $<.001$ \\
4 & Group $\times$ conflict in trial $n-1$ & 1 & 14054 & 38.4 & $<.001$ \\
5 & Group $\times$ conflict in trial $n$ & 1 & 14054 & 99.7 & $<.001$ \\
\hline
\end{tabular}

crease in ITs after an incompatible trial was greater when such trials were infrequent (26 vs. $11 \mathrm{msec}$ ). Since there were terms neither for the interaction between conflict in trials $n-1$ and $n$ nor for the interaction of these factors with conflict frequency, there is little evidence for such effects in the ITs.

\section{Movement Times}

Average MTs are also reported in Table 1, and the ANOVAs of the most parsimonious model are given in Table 4 . The model included terms for conflict frequency, conflict in trial $n-1$, conflict in trial $n$, the interaction between conflict frequency and conflict in trial $n$, and the interaction between conflict in trial $n-1$ and conflict in trial $n$. MTs were shorter overall in the frequent-conflict group (115 vs. $145 \mathrm{msec}$ ); in addition, whereas conflict in trial $n-1$ did not influence MTs, conflict in trial $n$ did lead to longer MTs (150 vs. $110 \mathrm{msec})$. The flanker was different between groups, with a stronger effect in the infrequent-conflict group (53 vs. $28 \mathrm{msec}$ ). Furthermore, there was an interaction of conflict in trial $n-1$ and trial $n$, with a reduced flanker effect after conflict in trial $n-1$ (36 vs. $45 \mathrm{msec}$ ). Since no term for a three-way interaction was included in the model, recency modulation did not differ between the groups.

\section{DISCUSSION}

In the present study, we investigated the interplay of conflict frequency and conflict recency effects in the flanker task. We replicated previous findings of reductions in the flanker effect after a directly preceding incompatible trial (i.e., the recency modulation; see, e.g., Ullsperger et al., 2005) and under conditions of globally increased frequency of incompatible trials (i.e., the frequency modulation; e.g., Gratton et al., 1992). Note that the recency effects obtained in the present study were low (i.e., 6 and $3 \mathrm{msec}$ for the infrequent- and frequent-conflict groups, respectively) relative to other studies (e.g., Gratton et al., 1992). Although studies on the recency modulation have differed in a number of procedural aspects, thus making comparisons difficult, it seems likely that the small effect size in our study results from our method for controlling the stimulus and response feature sequence (i.e., using four different stimuli as targets and flankers and eliminating all feature repetitions from the statistical analyses). In this light, it is interesting that using a similar method, Ullsperger et al. (2005, Experiment 2) observed a recency modulation of only $7 \mathrm{msec}$, which they considered to be a consequence of a low overall flanker effect - that is, averaged across compatible and incompatible predecessor trials - associated with their experimental makeup. This explanation cannot be applied to our data, since the overall flanker effect we obtained is comparable to those in other studies that found larger recency effects when feature sequence was not controlled. Thus, the small recency effects observed in the present study suggest that in the absence of feature sequence control, the size of attentional adjustment may be severely overestimated.

As predicted by models that assume one mechanism controlling both recency and frequency modulations (e.g., Botvinick et al., 2001), the recency effect was smaller under conditions of an overall higher proportion of incompatible trials. Nevertheless, this result cannot be taken as evidence for one-mechanism models, since the same prediction can be derived from models assuming two mechanisms, as long as the effect of one of the mechanisms depends on the effect of the other (see, e.g., Stürmer et al., 2002).

Analyzing ITs and MTs separately yielded additional important results. For one thing, a flanker interference effect was found in both response components, demonstrating that participants did not complete response selection before releasing the rest button. For another, RTs were generally greater under conditions of frequent conflict, an effect due entirely to delayed response initiation, whereas MTs were shorter when conflict frequency was high. This IT-MT trade-off suggests that frequent conflict prompted participants to perform a larger portion of response selection before releasing the rest button.

Whereas the conflict frequency modulation occurred in both response components, reduction of interference after a preceding conflict trial was confined to MTs. Although this result is consistent with the assumption of two mechanisms - associated with different time coursesunderlying the recency and frequency modulations, interpretation of these mechanisms is rendered difficult by the fact that response initiation in general was delayed in the frequent-conflict group. This delay makes it conceivable that the frequency modulation found during response initiation could reflect gradually increasing selectivity during stimulus processing that was unrelated to previous

Table 4 ANOVA Table for the Most Parsimonious Movement Time Model

\begin{tabular}{llrrrr}
\hline \multicolumn{1}{c}{ Term } & $d f_{\text {num }}$ & $d f_{\text {den }}$ & \multicolumn{1}{c}{$F$} & \multicolumn{1}{c}{$p$} \\
\hline 1 Group & 1 & 14054 & 52.2 & .028 \\
2 Conflict in trial $n-1$ & 1 & 14054 & 92.6 & .396 \\
3 & Conflict in trial $n$ & 1 & 14054 & 509.1 & $<.001$ \\
4 Group $\times$ conflict in trial $n$ & 1 & 14054 & 38.4 & $<.001$ \\
5 & Conflict in trial $n-1 \times$ conflict in trial $n$ & 1 & 14054 & 99.7 & .012 \\
\hline
\end{tabular}


conflict, such as more advanced perceptual target-flanker segregation, and that conflict-based selectivity adjustment may not become effective before later processing stages, thereby affecting MTs only. To test this possibility, we calculated the flanker effect for IT quintiles (cf. Hommel, 1997). If anything, in this analysis the flanker effect increased with increasing IT $(8,23,38,50$, and $53 \mathrm{msec}$ for the 1st to 5 th quintiles in the infrequent-conflict group; $14,20,24,28$, and $34 \mathrm{msec}$ for each of these quintiles in the frequent-conflict group), thus casting doubt on the idea that the reduced flanker effect for the frequentconflict group was a simple by-product of an overall delay in response initiation.

An open question is why the recency and frequency modulations, which interacted in RTs, did not interact in MTs. This lack of consistency might result from the exploratory data analysis strategy that we applied for the ITs and MTs. On the other hand, it might result from a very small effect in ITs that combined with a small effect in MTs to create a detectable effect in RTs.

Taken together, our data support models featuring two dependent mechanisms. It is important to note, however, that the study does not allow us to decide what these two mechanisms are. On one hand, frequency modulation could result from flanker-response priming (cf. Stürmer et al., 2002). Increasing the frequency of conflict trials implies that flankers will predominantly co-occur with an incompatible response (i.e., a response different from the one that would be required if the flanker character occurred as the target). Therefore, it is conceivable that learned flanker-response associations - depending for their strength on the number of co-occurrences-could result in priming of an incorrect response in compatible trials and of the correct response in incompatible trials, thereby reducing response conflict and (as a behavioral measure of response conflict) the flanker effect. Assuming that the recency modulation depends on response conflict, less modulation would be needed under conditions of frequent conflict. On the other hand, any mechanism that decreases the conflict exerted by an incompatible stimulus (on a directly preceding trial) should also lead to a weaker recency modulation. That is, two attentional mechanisms are possible in which one mechanism enforces a task set (strategic adaptation to frequent conflict), whereas the other responds to changes in the need for cognitive control on a trial-by-trial basis. This situation would be comparable to Ridderinkhof's (2002) notion of micro- and macro-adjustments.

Future research is needed in order to clarify the circumstances under which modulation of interference effects represents conflict-related adjustment per se, as well as to further characterize such adjustments in terms of the stage of information processing in which they become effective. Decomposing RTs into ITs and MTs, as was done in this study, might be a useful approach to these goals, since it allows for a more fine-grained examination of cognitive processes, and even has the potential to provide some information on the time course of these processes.

\section{AUTHOR NOTE}

S.P. is supported by the (German) Federal Ministry of Education and Research (BMBF). We thank Tom Busey, Anne Hillstrom, and Bernhard Hommel for valuable comments on an earlier version of this article. Correspondence concerning this article should be addressed to S. Purmann, Ruprecht Karls University Heidelberg, Hauptstraße 47-51, 69117 Heidelberg, Germany (e-mail: sascha.purmann@psychologie .uni-heidelberg.de).

\section{REFERENCES}

AKAIKE, H. (1973). Information theory and the maximum likelihood principle. In B. N. Petrov \& F. Csáki (Eds.), Second International Symposium on Information Theory (pp. 267-281). Budapest: Akadémiai Kiadó.

Botvinick, M. M., Braver, T. S., Barch, D. M., Carter, C. S., \& CoHen, J. D. (2001). Conflict monitoring and cognitive control. Psychological Review, 108, 624-652.

ERIKSEN, B. A., \& ERIKSEN, C. W. (1974). Effects of noise letters upon the identification of a target letter in a nonsearch task. Perception \& Psychophysics, 16, 143-149.

Gratton, G., Coles, M. G. H., \& Donchin, E. (1992). Optimizing the use of information: Strategic control of activation of responses. Journal of Experimental Psychology: General, 121, 480-506.

Hommel, B. (1994). Spontaneous decay of response-code activation. Psychological Research, 56, 261-268.

Hommel, B. (1997). Interactions between stimulus-stimulus congruence and stimulus-response compatibility. Psychological Research, 59, 248-260.

Hommel, B. (1998). Event files: Evidence for automatic integration of stimulus-response episodes. Visual Cognition, 5, 183-216.

Hommel, B., Proctor, R. W., \& Vu, K.-P. L. (2004). A feature-integration account of sequential effects in the Simon task. Psychological Research, 68, $1-17$

Kerns, J. G., Cohen, J. D., MacDonald, A. W., III, Cho, R. Y., Stenger, V. A., \& Carter, C. S. (2004). Anterior cingulate conflict monitoring and adjustments in control. Science, 303, 1023-1026.

Logan, G. D., \& ZBrodoff, N. J. (1979). When it helps to be misled: Facilitative effects of increasing the frequency of conflicting stimuli in a Stroop-like task. Memory \& Cognition, 7, 166-174.

Pinheiro, J. C., \& Bates, D. M. (2000). Mixed-effects models in $S$ and $S$-PLUS. New York: Springer.

R Development Core Team (2007). R: A language and environment for statistical computing. Vienna: R Foundation for Statistical Computing. Available at www.r-project.org.

RIDDERINKHOF, K. R. (2002). Micro- and macro-adjustments of task set: Activation and suppression in conflict tasks. Psychological Research, 66, 312-323.

Simon, J. R., Craft, J. L., \& Small, A. M., JR. (1971). Reactions toward the apparent source of an auditory stimulus. Journal of Experimental Psychology, 89, 203-206.

Stroop, J. R. (1935). Studies of interference in serial verbal reactions. Journal of Experimental Psychology, 18, 643-662.

Stürmer, B., Leuthold, H., Soetens, E., Schröter, H., \& SomMER, W. (2002). Control over location-based response activation in the Simon task: Behavioral and electrophysiological evidence. Journal of Experimental Psychology: Human Perception \& Performance, 28, 1345-1363.

Ullsperger, M., Bylsma, L. M., \& Botvinick, M. M. (2005). The conflict adaptation effect: It's not just priming. Cognitive, Affective, \& Behavioral Neuroscience, 5, 467-472.

(Manuscript received September 19, 2007; revision accepted for publication October 10, 2008.) 